\section{Unique solution for Zygomatic dental implant treatment}

Complex bone augmentation procedures are often stretched to their limits in cases of high-risk patients, poor compliance and extreme bone resorption. Zygoma dental implants offer both patients and clinicians an alternative if surgical intervention in the upper jaw is indicated.

The new SZ-75 handpiece has been developed by W\&H in collaboration with leading Zygoma dental implantologists. It is specifically designed to enable ultra-safe Zygomatic dental implant surgery and placement, with an innovative chucking system that makes this contra-angle handpiece the only one of its kind.

Easy access to the treatment site Zygoma implants are inserted into the zygomatic bone at a specific angle. It is crucial that the implant is accurately positioned to ensure success. The new W\&H SZ-75 handpiece makes Zygomatic

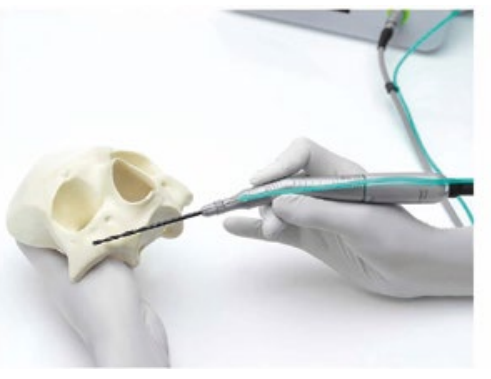

implant procedures easier to perform, as the ergonomic angulation of the instrument facilitates optimal access to the treatment site. This enables the clinician to work for extended periods of time with minimum risk of strain or fatigue.

\section{Ultra-safe to use}

Conventional surgical handpieces do not always clutch Zygoma implant burs securely. The SZ-75 from W\&H is the world's first 20:1 contra-angle handpiece to combat this issue with a unique chucking system that is optimised for Zygomatic dental implant surgery. This innovative solution makes the SZ-75 ultra-safe to use, whilst ensuring highly accurate concentricity during application. In addition, the handpiece can be used with standard dental implant burs and applied universally in surgery.

\section{Efficient spray cooling}

The new W\&H Zygoma handpiece also features an external spray channel that expels coolant close to the tip of the instrument to ensure efficient cooling of the treatment site, thus eliminating the need for manual cooling.

To find out more visit www. wh.com/en_uk, call 01727874990 or email office.uk@wh.com.

\title{
Our promise stands strong
}

At Colosseum Dental UK, we are committed to providing a great place to work for all of our clinicians. That's why we make promises to our associate dentists and we do everything we can to deliver on each one.

$\rightarrow$ We will pay you 1/12th for the first 6 months as we know it takes time to settle in

$\rightarrow$ Excellent working facilities

$\rightarrow$ The highest standards of clinical governance and expert support

$\rightarrow$ Access to ongoing CPD

$\rightarrow$ Mentoring opportunities

$\rightarrow$ Qualified dental nurses

$\rightarrow$ Access to a wide range of consumables

$\rightarrow$ Work with the lab of your choice

(DAMAS registered)

$\rightarrow$ Well-established patient lists

\section{$\rightarrow$ New Pastelli uniforms \\ $\rightarrow$ Equipment repairs fixed in a day.}

So, if you're looking for a new position where you will feel valued, listened to, challenged and supported, consider joining Colosseum Dental UK. For more information about Colosseum Dental, please visit www.colosseumdental.co.uk/careers.

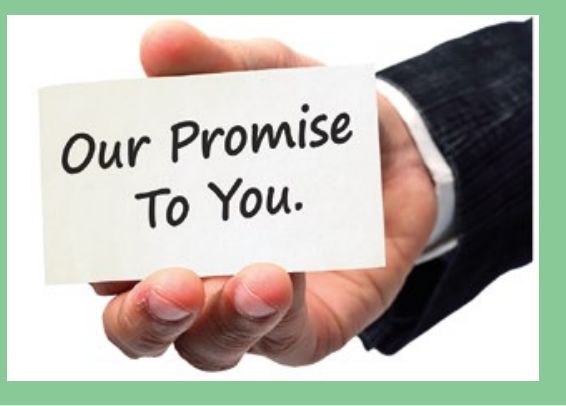

Still there for patients and professionals

Despite all the challenges of the past year, Rodericks Dental remains committed to delivering excellent patient care from all of its practices across England and Wales. Processes have changed, protocols updated and training delivered to ensure that all members of our dental teams have the knowledge and confidence to continue working in a safe and effective way.

To facilitate quality patient care, we offer a choice of quality materials in our practices. We also continue to support all our professionals. There is always someone to turn to for help or guidance, from Clinical Advisors to Education Supervisors, practice mangers and senior directors.

For more information on the career opportunities available at Rodericks visit www.rodericksdentalcareers.co.uk or contact Ashley Lillyman at recruitment@ rodericksdental.co.uk

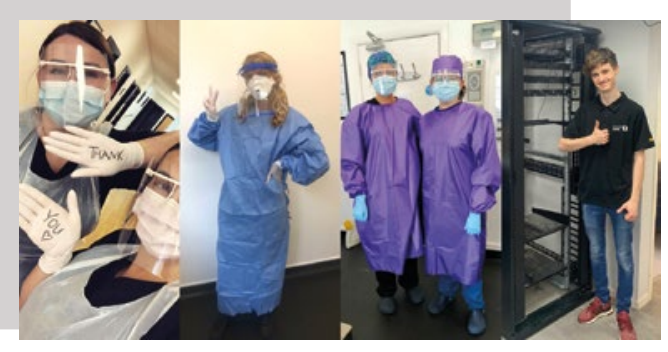

The right choice for referrals

At EndoCare, our philosophy is ensuring that every patient gets the help they need, regardless of the complexity of the case.

With decades of experience behind us, the team are all trained to perform only the most up-to-date endodontic treatments and techniques, meaning that we can offer patients practically pain-free treatment and solve even the most complex cases.

When you refer to EndoCare you can rest assured that you will return your patients to your care with comprehensive details of the treatment they have received and our suggested next steps.

Get a helping hand and give your patients the specialist care they need by contacting EndoCare on 02072240999 or by visiting www.endocare.co.uk. 